\title{
Transgenic Expression of a Dominant-Negative ASIC3 Subunit Leads to Increased Sensitivity to Mechanical and Inflammatory Stimuli
}

\author{
Jeffrey S. Mogil, ${ }^{1}$ Nicole M. Breese, ${ }^{2}$ Marie-France Witty, ${ }^{3}$ Jennifer Ritchie, ${ }^{1}$ Marie-Line Rainville, ${ }^{3}$ Ariel Ase, ${ }^{3}$ \\ Naima Abbadi, ${ }^{3}$ Cheryl L. Stucky, ${ }^{2}$ and Philippe Séguéla ${ }^{3}$ \\ ${ }^{1}$ Department of Psychology and Centre for Research on Pain, McGill University, Montreal, Quebec, Canada H3A 1B1, ${ }^{2}$ Department of Cell Biology, \\ Neurobiology, and Anatomy, Medical College of Wisconsin, Milwaukee, Wisconsin 53226, and ${ }^{3}$ Montreal Neurological Institute, Department of Neurology \\ and Neurosurgery, and Centre for Research on Pain, McGill University, Montreal, Quebec, Canada H3A 2B4
}

\begin{abstract}
Molecular and behavioral evidence suggests that acid-sensing ion channels (ASICs) contribute to pain processing, but an understanding of their precise role remains elusive. Existing ASIC knock-out mouse experiments are complicated by the heteromultimerization of ASIC subunits. Therefore, we have generated transgenic mice that express a dominant-negative form of the ASIC3 subunit that inactivates all native neuronal ASIC-like currents by oligomerization. Using whole-cell patch-clamp recordings, we examined the response properties of acutely isolated dorsal root ganglion neurons to protons ( $\mathrm{pH} 5.0$ ). We found that whereas $33 \%$ of the proton-responsive neurons from wild-type mice exhibited an ASIC-like transient response, none of the neurons from the transgenic mice exhibited a transient inward current. Capsaicin-evoked responses mediated by the TRPV1 receptor were unaltered in transgenic mice. Adult male wild-type and transgenic mice were subjected to a battery of behavioral nociceptive assays, including tests of thermal, mechanical, chemical/inflammatory, and muscle pain. The two genotypes were equally sensitive to thermal pain and to thermal hypersensitivity after inflammation. Compared with wild types, however, transgenic mice were more sensitive to a number of modalities, including mechanical pain (von Frey test, tail-clip test), chemical/inflammatory pain (formalin test, $0.6 \%$ acetic acid writhing test), mechanical hypersensitivity after zymosan inflammation, and mechanical hypersensitivity after intramuscular injection of hypotonic saline. These data reinforce the hypothesis that ASICs are involved in both mechanical and inflammatory pain, although the increased sensitivity of transgenic mice renders it unlikely that they are direct transducers of nociceptive stimuli.
\end{abstract}

Key words: acid-sensing ion channels; sensory neurons; dorsal root ganglia; pain; mutant; hyperalgesia

\section{Introduction}

Since the discovery of proton-activated conductances in 1980 (Krishtal and Pidoplichko, 1980) and the recent cloning of the amiloride-sensitive, acid-sensing ion channels (ASICs) (Waldmann et al., 1997), many have suspected that proton-gated channels might play a role in the transduction of pain. The ASICs can be found in all subtypes of dorsal root ganglion (DRG) sensory neurons (Alvarez de la Rosa et al., 2002), and many pathological conditions in peripheral tissues involve acidosis, which activates nociceptors (Reeh and Steen, 1996). Evidence suggests that ASICs might play a role in the transduction of inflammatory

\footnotetext{
Received May 19, 2005; revised Sept. 8, 2005; accepted Sept. 9, 2005.

This work was supported by Antalium Inc. (P.S.), Canadian Institutes of Health Research Grant MOP-49601 (P.S.), and National Institutes of Health Grants DA15191 (J.S.M.) and NS40538 (C.L.S.). J.S.M. holds a Canada Research Chair, and P.S. is a Killam Scholar. We gratefully acknowledge the following collaborators for helpful discussions and technical assistance during this project: Kazimierz Babinski and Dr. Stefano Catarsi (Antalium Inc., Montreal, Quebec, Canada), David Reese and Dominique Blais (Montreal Neurological Institute, McGill University, Montreal, Quebec, Canada), Drs. François Besnard and Stéphane Renard (Sanofi-Synthelabo France, Paris, France), and Laura Pauers (Medical College of Wisconsin, Milwaukee, WI).

Correspondence should be addressed to Dr. Philippe Séguéla, Montreal Neurological Institute, 3801 University, Office 778, Montreal, Quebec, Canada H3A 2B4. E-mail: philippe.seguela@mcgill.ca.

D0I:10.1523/JNEUROSCI.2019-05.2005

Copyright $\odot 2005$ Society for Neuroscience $\quad$ 0270-6474/05/259893-09\$15.00/0
}

pain, because ASIC expression is facilitated by inflammation (Voilley et al., 2001) and pro-inflammatory algogens (Mamet et al., 2002). Furthermore, there is evidence for the involvement of ASICs in noxious mechanosensation (Price et al., 2001; Sluka et al., 2003), and members of the same receptor superfamily serve this function in lower organisms (O'Hagan et al., 2005). However, establishing the true role of ASICs in pain processing has been complicated by a number of factors, including the rapid desensitization of ASICs, their unknown subunit composition, the lack of selective pharmacological tools, and the contribution of TRPV1 receptor and/or background $\mathrm{K}^{+}$channels in DRG neurons as transducers of acidosis-related nociception (Tominaga et al., 1998; Baumann et al., 2004).

The involvement of ASICs in pain sensation has been investigated recently using mice deficient in specific ASIC subunits. Studies with ASIC2 knock-outs (in which both ASIC2a and ASIC2b splice variants have been disrupted) suggest that this channel has no involvement in mechanical sensation in the noxious range (Price et al., 2000; Drew et al., 2004; Roza et al., 2004), although the mice have yet to be tested behaviorally. Three separate studies have evaluated the behavioral phenotype of ASIC3 knock-out mice, with disparate results. Price et al. (2001) ob- 
served a small but significant increase in sensitivity to mechanical stimulation after hindpaw inflammation (i.e., mechanical hypersensitivity) and a reduced development of mechanical hypersensitivity after injection of acid ( $\mathrm{pH} 4$ saline) into the gastrocnemius muscle. Sluka et al. (2003) later confirmed that the hypersensitivity induced by acid injection in muscle is specific to ASIC3, because ASIC1 knock-outs displayed mechanical hypersensitivity equivalent to that in wild-type mice. In contrast, Chen et al. (2002) observed an increased sensitivity to thermal, mechanical, and acid stimuli in ASIC3 knock-outs (at higher stimulus intensities only) but no alterations in hypersensitivity after capsaicin or carrageenan.

ASICs are known to form heteromultimers in native DRG neurons (Benson et al., 2002). Therefore, experiments using mice deficient in single ASIC subunits may not be well suited to gleaning the true role of these genes in pain processing. We have adopted a different strategy in which we have generated transgenic mice that express a dominant-negative form of the ASIC3 subunit, which inactivates all native neuronal ASIC currents by oligomerization. We have used extensive behavioral assays and electrophysiological recordings to characterize the sensory phenotype of these transgenic animals.

\section{Materials and Methods \\ ASIC clones, mutants, and transgenesis}

Rodent ASIC1a, ASIC2a, and ASIC3 subunits were cloned using reverse transcription (RT)-PCR. The human ASIC3 (hASIC3) clone was described previously (Babinski et al., 1999). Single mutants of hASIC3 were generated using the Quikchange method (Stratagene, La Jolla, CA). The hASIC3 G439R subunit cDNA was subcloned downstream of the neuron-specific promoter of the human neurofilament light chain gene (Charron et al., 1995), kindly provided by J. P. Julien (Laval University, Quebec City, Quebec, Canada), and upstream of the bovine growth hormone poly(A) sequence to increase mRNA stability (see Fig. 2 A). Transgenic FVB strain mice were generated by pronuclear microinjection of linearized DNA in fertilized oocytes at the core transgenesis unit of McGill University. Homozygous transgenic animals were first selected using Southern blots and genomic PCR with transgene-specific primers (sequences GATGGCTGGCAACTAGAAGG and ATGAGACCGTGGAGCAGAAG), then confirmed by breeding using the homocheck method. Expression of the transgene coding for mutant ASIC3 G439R subunits in the central and peripheral nervous systems was verified using (1) Northern blots from total brain and kidney, with a probe corresponding to the artificial hybrid sequence ASIC3-bGH poly(A), and (2) RTPCR for detection of exogenous hASIC3 mRNA in total brain and DRG of adult mice.

\section{Electrophysiological recordings in oocytes and human embryonic kidney 293 cells}

Oocytes used for the screening of candidate mutations were surgically removed from Tricaine-anesthetized female Xenopus laevis frogs and incubated with calcium-free Barth's solution containing $1 \mathrm{mg} / \mathrm{ml}$ type I collagenase at room temperature for $2 \mathrm{~h}$ under vigorous agitation. Stage $\mathrm{V}$ and VI oocytes were then defolliculated manually before intranuclear microinjection of 5-20 ng of supercoiled plasmid coding for wild-type/ mutant hASIC3 subunits. After injection, oocytes were incubated with Barth's solution containing $1.8 \mathrm{mM} \mathrm{CaCl}_{2}$ at $19^{\circ} \mathrm{C}$ for $24-72 \mathrm{~h}$ before electrophysiological recordings.

Two-electrode voltage-clamp recordings $\left(V_{\mathrm{H}}=-60 \mathrm{mV}\right)$ were performed using glass pipettes (1-3 M $\Omega$ ) filled with $3 \mathrm{M} \mathrm{KCl}$ solution. Oocytes were placed in a recording chamber and superfused at a flow rate of $10-12 \mathrm{ml} / \mathrm{min}$ with Ringer's solution, $\mathrm{pH} 7.4$ or 5.0 , containing the following (in mM): $115 \mathrm{NaCl}, 5 \mathrm{NaOH}, 2.5 \mathrm{KCl}, 1.8 \mathrm{CaCl}_{2}$, and 10 HEPES. Membrane currents (DC, $1 \mathrm{kHz}$ ) were recorded using a Warner (Hampden, CT) OC-725B amplifier and digitized at $500 \mathrm{~Hz}$.

Human embryonic kidney 293 (HEK293) or COS-7 cells were cultured in DMEM and 10\% heat-inactivated fetal bovine serum (Invitro- gen, San Diego, CA) containing penicillin and streptomycin supplemented with Geneticin $(250 \mu \mathrm{g} / \mathrm{ml})$. Cells were transfected with Polyfect (Qiagen, Chatsworth, CA) or Fugene 6 (Roche Diagnostics, Laval, Quebec, Canada) according to the manufacturer's protocols. Transfected cells were used for electrophysiological recordings $24-48 \mathrm{~h}$ after transfection. Whole-cell patch-clamp recordings $\left(V_{\mathrm{H}}=-60 \mathrm{mV}\right)$ were performed using pipettes filled with internal solution, $\mathrm{pH}$ 7.2, containing (in mM) $120 \mathrm{~K}$-gluconate, $1 \mathrm{MgCl}_{2}, 4 \mathrm{NaOH}$, and $10 \mathrm{HEPES}$. Drugs were applied using a Warner SF-77B fast perfusion system at a rate of $1 \mathrm{ml} /$ min. The perfusion solution, $\mathrm{pH} 7.4$ or 5.0, comprised the following (in mM): $145 \mathrm{NaCl}, 5 \mathrm{NaOH}, 3 \mathrm{KCl}, 1 \mathrm{MgCl}_{2}, 0.9 \mathrm{CaCl}_{2}$, and 10 HEPES. Membrane currents (DC, $200 \mathrm{~Hz}$ ) were recorded using an Axopatch 200B amplifier and digitized at $500 \mathrm{~Hz}$. All experiments were performed at room temperature.

\section{Electrophysiological recordings in wild-type and transgenic DRG neurons}

Mice were anesthetized with isoflurane and killed by cervical dislocation, and all lumbar (L1-L6) DRGs were removed. A total of 15 mice (8 transgenic, 7 wild type) were used for these experiments. The ganglia were incubated with $1 \mathrm{mg} / \mathrm{ml}$ collagenase IV (Sigma, St. Louis, MO) and $0.05 \%$ trypsin (Sigma) for $40 \mathrm{~min}$ each at $37^{\circ} \mathrm{C}$ and dissociated into single cells by passing though flame-constricted Pasteur pipettes of decreasing diameter. The cells were washed and resuspended in DMEM/Ham's F-12 medium containing $10 \%$ heat-inactivated horse serum, $20 \mathrm{~mm}$ glutamine, $0.8 \%$ glucose, $100 \mathrm{U}$ of penicillin, and $100 \mu \mathrm{g} / \mathrm{ml}$ streptomycin (Invitrogen). Cells were plated onto glass coverslips coated with poly-Llysine and maintained at $37^{\circ} \mathrm{C}, 5 \% \mathrm{CO}_{2}$ for $\leq 9 \mathrm{~h}$. No exogenous nerve growth factor (NGF) was added to the medium, because NGF has been shown to alter the response properties of small-diameter neurons to capsaicin and heat within minutes (Shu and Mendell, 2001). Whole-cell recordings from the cell soma were performed between 1.5 and $9 \mathrm{~h}$ after isolation. Experiments performed within this acute time frame allow neurons to adhere to the coverslip but avoid many artifacts that occur in neurons cultured for longer time periods. The acute time frame also avoids the loss of capsaicin sensitivity that occurs in adult rat DRG neurons cultured without NGF (half-life of 3 d) (Winter et al., 1988).

Whole-cell recordings were made from neurons using fire-polished glass electrodes (2-6 $\mathrm{M} \Omega$ resistance) pulled from borosilicate glass on a micropipette puller (P-87; Sutter Instruments). The recording chamber was superfused continuously with solution containing the following (in $\mathrm{mm}$ ): $150 \mathrm{NaCl}, 5.6 \mathrm{KCl}, 2 \mathrm{CaCl}_{2}, 1 \mathrm{MgCl}_{2}, 10 \mathrm{HEPES}$, and 8 glucose, $\mathrm{pH}$ 7.4 adjusted with $\mathrm{NaOH}$, osmolarity of $320 \mathrm{mOsm}$. Electrodes were filled with solution containing the following (in $\mathrm{mm}$ ): $135 \mathrm{KCl}, 10 \mathrm{NaCl}, 1$ $\mathrm{MgCl}_{2}, 1$ EGTA, 10 HEPES, and 2.5 NaATP 2 , pH 7.2 adjusted with $\mathrm{KOH}$, osmolarity of $290 \mathrm{mOsm}$. All solutions were made fresh daily, filtered just before use, and used at room temperature $\left(22-24^{\circ} \mathrm{C}\right)$. Neurons were viewed with a Nikon (Mississauga, Ontario, Canada) TE200 inverted microscope, and the soma size was estimated by calculating the mean of the longest and shortest diameters using a calibrated eyepiece reticle. Neurons of all soma sizes were recorded.

For chemical tests with protons and capsaicin, solutions were applied locally and rapidly (10 s duration) to the neuron of interest using 28 gauge nonmetallic needles (inner diameter, $0.25 \mathrm{~mm}$; World Precision Instruments, Sarasota, FL). The tip of each needle was placed $50 \mu \mathrm{m}$ from the cell soma using a manipulator (Narishige, Tokyo, Japan), and the gravity-fed solutions were controlled manually by switching one-way stopcock valves (Cole-Parmer, Vernon Hills, IL). For experiments with capsaicin, a $1 \mu \mathrm{M}$ concentration of capsaicin (in HEPES buffer) was prepared daily from a $10 \mathrm{~mm}$ capsaicin stock solution dissolved in 1-methyl-2-pyrrolidinone (Sigma), a solvent that has no effect on the physiology of sensory neurons (Breese et al., 2005). For stimulation with protons, the HEPES in the extracellular buffer was replaced with 2-[Nmorpholino] ethanesulfonic acid, and the $\mathrm{pH}$ was adjusted to 5.0 with $\mathrm{HCl}$. Because transient ASIC-like responses are reproducible in magnitude after multiple applications of pH 5.0 (N. M. Breese and C. L. Stucky, unpublished observations), two to three neurons were tested per coverslip with at least a 7 min wash between proton tests. Because $1 \mu \mathrm{M}$ capsaicin desensitizes subsequent capsaicin-evoked inward currents for at 
least $10 \mathrm{~min}$ (Breese and Stucky, unpublished observations), only one neuron per coverslip was tested with capsaicin.

Membrane voltage was clamped using an EPC-9 amplifier run by Pulse software (version 8.65; HEKA Electronic, Lambrecht, Germany). Data were sampled at $10 \mathrm{kHz}$. The membrane potential was held at -70 $\mathrm{mV}$. Neurons were included in our analysis only if they formed seals $>1.5$ $\mathrm{G} \Omega$, had resting membrane potentials more negative than $-45 \mathrm{mV}$ in current-clamp mode, and had an input resistance $>100 \mathrm{M} \Omega$ after wholecell configuration was established. The size of the cell capacitance transients was monitored throughout the recording and did not change by $>10 \%$. Pipette and cell capacitance were compensated using the computer-controlled circuitry. Series resistance was compensated by $70-90 \%$. The voltage-clamp recording mode was used to measure the inward current evoked by chemical stimuli, and the magnitude of inward current was determined using PulseFit software. Neurons were considered proton or capsaicin sensitive if either chemical elicited an inward current $>100 \mathrm{pA}$ in peak amplitude. Proton responses were classified as transient (rapidly inactivating within 1-2 s) or sustained (slow inactivation $>20 \mathrm{~s}$ ) as described by Dirajlal et al. (2003). To correct for differences in soma size, all inward current values are expressed as a function of cell capacitance $(\mathrm{pA} / \mathrm{pF})$. For statistical measures, groups were compared using Fisher's exact test, an unpaired two-tailed $t$ test, or one-way ANOVA, as appropriate, using InStat (Graph Pad, San Diego, CA).

\section{Animals in behavioral experiments}

Subjects were naive adult (6-12 weeks old) male mice of the following genotypes: wild-type $\mathrm{FVB} / \mathrm{J}$ and homozygous transgenic $\mathrm{FVB} / \mathrm{J}$ hASIC3DN $\left(\mathrm{F}_{2}-\mathrm{F}_{4}\right)$. In one experiment, C57BL/6J mice (The Jackson Laboratory, Bar Harbor, ME) were also used. Mice were transferred from one vivarium on the McGill University campus to another at $\geq 5$ weeks of age and habituated to the new vivarium for at least 1 week before testing commenced. Mice were housed with others of the same genotype (two to four mice per cage) in a temperature-controlled $\left(20 \pm 1^{\circ} \mathrm{C}\right)$ environment and with ad libitum access to food (Harlan Teklad 8604) and tap water.

\section{Algesiometric assays}

Behavioral testing was conducted between 8:00 A.M. and 5:00 P.M., and genotypes were fully counterbalanced. All animals were habituated to the testing room, immediately adjacent to the vivarium, for at least $30 \mathrm{~min}$ before testing commenced.

Mice were tested by a single highly experienced experimenter on a battery of algesiometric assays, most described in considerable detail in the studies by Mogil et al. (1999) and Lariviere et al. (2002). To reduce subject requirements for reasons of practicality, acute assays were performed on the same set of subjects, in the following order, with at least $2 \mathrm{~d}$ separating each test: tail-withdrawal test, hot-plate test, paw-withdrawal test, von Frey test, and tail-clip test. Pilot studies have shown that these acute assays are minimally affected by previous testing on other assays within the group (J. Ritchie, S. G. Sotocinal, M. L. Chanda, and J. S. Mogil, unpublished data). These subjects were then tested with either the abdominal constriction test or the formalin test. Separate sets of naive subjects were used for the zymosan and intramuscular hypotonic saline assays. When necessary because of higher test-related variability, additional subjects were added, but always in a counterbalanced manner according to genotype. A brief description of these assays, in alphabetical order, is provided below.

Abdominal constriction test. After habituation to individual Plexiglas observation chambers, mice were given intraperitoneal injections (10 $\mathrm{ml} / \mathrm{kg}$ ) with $0.3,0.6$, or $0.9 \%$ acetic acid $(n=4-11$ per genotype per concentration), and the number of lengthwise abdominal constrictions ("writhes") were counted for $30 \mathrm{~min}$.

Formalin test. Mice ( $n=8-10$ per genotype) were habituated in individual Plexiglas cylinders ( $15 \mathrm{~cm}$ diameter, $22.5 \mathrm{~cm}$ high) placed atop a glass surface suspended over high-resolution video cameras. All subjects were then given a subcutaneous injection of $5 \%$ formalin into the plantar right hindpaw (20 $\mu$ l volume) and videotaped digitally for $60 \mathrm{~min}$ after the formalin injection. Videotape observations were later sampled for $5 \mathrm{~s}$ at $1 \mathrm{~min}$ intervals, and the presence or absence of right hindpaw licking/ biting in that $5 \mathrm{~s}$ period was scored. The acute phase of the formalin test was defined as $0-10 \mathrm{~min}$ after injection, and the tonic phase as $10-60$ min after injection. Data are presented as the percentage of samples in each phase in which licking/biting was detected. We have demonstrated previously the high accuracy of the sampling strategy in our hands (Chesler et al., 2003).

Hot-plate test. Mice were placed within a transparent Plexiglas cylinder ( $15 \mathrm{~cm}$ diameter, $22.5 \mathrm{~cm}$ high) on a metal surface maintained at 50.0 or $53.0^{\circ} \mathrm{C}\left( \pm 0.2^{\circ} \mathrm{C}\right)(n=11-23$ per genotype per temperature $)$. The latency to respond with a hindpaw lick or shake/flutter, whichever came first, was measured to the nearest $0.1 \mathrm{~s}$ with a stopwatch. This test was only performed once, because repeated testing leads to systematic latency alterations (Wilson and Mogil, 2001).

Intramuscular hypotonic saline test. We followed very closely the protocol of Sluka et al. (2003). All mice ( $n=6-7$ per genotype) were tested for baseline mechanical sensitivity using the \#5 von Frey fiber $(0.1 \mathrm{~g}$; Stoelting, Wood Dale, IL) applied five times to each hindpaw. Immediately thereafter, they were given an injection of $\mathrm{pH} 4$ saline (20 $\mu \mathrm{l}$ volume) into the right gastrocnemius muscle. Mice were retested for mechanical sensitivity on both hindpaws $4 \mathrm{~h}, 24 \mathrm{~h}$, and $4 \mathrm{~d}$ after this injection. Immediately after testing on day 4 , all mice were given a second injection of $\mathrm{pH} 4$ saline into the same muscle. Mice were retested for mechanical sensitivity on both hindpaws $1,2,4$, and $8 \mathrm{~d}$ after the second injection. Data are presented as the number of withdrawal responses (of five applications) for each hindpaw at each time point.

Paw-withdrawal test. Mice were placed on a 3/16 inch thick glass floor within small Plexiglas cubicles, and a focused high-intensity projector lamp beam was shone from below onto the mid-plantar surface of the hindpaw (Hargreaves et al., 1988). The commercial device (IITC model 336 ) was set to either $10 \%$ intensity or $20 \%$ intensity $(n=10-12$ per genotype per intensity). Latency to withdraw from the stimulus was measured to the nearest $0.1 \mathrm{~s}$. Mice were tested at four time points separated by at least $30 \mathrm{~min}$; at each time point, each hindpaw was tested twice, separated by at least $30 \mathrm{~s}$. The data presented are the grand means of the 16 separate latency determinations.

Tail-clip test. All mice ( $n=27-31$ per genotype) were lightly restrained in a cloth/cardboard holder, and an alligator clip with rubber cuffs around the teeth of each jaw (exerting $500 \mathrm{~g}$ of force) was applied to the tail $1 \mathrm{~cm}$ from the base. The mouse was immediately removed from the holder, and the latency to lick, bite, grab, or bring the nose to within $1 \mathrm{~cm}$ of the clip was measured with a stopwatch to the nearest $0.1 \mathrm{~s}$, after which the clip was removed immediately. This test was only performed once, because repeated testing leads to systematic latency alterations (S. B. Smith and Mogil, unpublished data).

Tail-withdrawal test. While lightly restrained in a cloth/cardboard holder, the distal half of the mouse's tail was dipped into a bath of water thermostatically controlled at 47.0 or $49.0^{\circ} \mathrm{C}\left( \pm 0.1^{\circ} \mathrm{C}\right)(n=10-13$ per genotype per temperature). Latency to respond to the heat stimulus by vigorous flexion of the tail was measured. Mice were tested at four time points separated by $30 \mathrm{~min}$; at each time point, two latency determinations (separated by $\geq 20 \mathrm{~s}$ ) were made and averaged.

von Frey test. For assessment of more accurate mechanical sensitivity thresholds, mice were tested on the von Frey test using the up-down staircase method (Chaplan et al., 1994). Mice ( $n=8-13$ per genotype) were placed on a metal mesh floor within small Plexiglas cylinders, and a set of eight calibrated von Frey fibers (ranging from 0.007 to $1.40 \mathrm{~g}$ of force) were applied to the plantar surface of the hindpaw. The threshold force required to elicit withdrawal of the foot (median 50\% foot withdrawal) was determined. Data from both the left and right hindpaws were averaged together.

Zymosan hypersensitivity. Mice were assessed for their baseline sensitivity on the radiant heat paw-withdrawal test (intensity setting, 10\%; four baselines on each hindpaw separated by $15 \mathrm{~min}$ ) or to the application of three von Frey fibers (\#5, \#7, and \#9 of the Stoelting set) applied six times to each hindpaw. Separate mice were used for each assay $(n=$ 5-13 per genotype per assay). Immediately after baseline measurements, all mice were given subcutaneous injections into the right hindpaw, with a $1.0 \mathrm{mg} / \mathrm{ml}$ solution of zymosan (20 $\mu$ l injection volume) (Meller and Gebhart, 1997). Mice were retested for thermal or mechanical sensitivity of both hindpaws at 60, 120, 180, 240, 300, and 360 min after injection. 
Behavioral data analysis

In the behavioral experiments, data from three mice were excluded after they were identified as statistical outliers (Studentized residual, >3.0). In most assays, genotype differences were evaluated using Student's $t$ test (two-way), with $\alpha=$ 0.05 . In the zymosan hypersensitivity and intramuscular hypotonic saline tests, time course data were analyzed by one-between (genotype), onewithin (time) repeated-measures ANOVAs, followed, when appropriate, by (Sidak-corrected) post hoc tests for repeated measures (Systat version 10.2; SPSS, Chicago, IL) and Student's $t$ tests to establish genotype differences at particular time points.

\section{Results}

Mutant ASIC3 subunit has

\section{dominant-negative properties}

We observed that the single mutation G439R, located in a conserved domain of the pre-transmembrane 2 (TM2) region of all known ASIC subunits, confers insensitivity to protons to the hASIC3 subunit as well as dominant-negative properties when coexpressed with wild-type human or rat ASIC3 in Xenopus oocytes (data not shown) or with wild-type rat ASIC3 in transfected HEK293 cells $(8.7 \pm 1.4$ vs $1.2 \pm 0.4 \mathrm{nA}$ at a ratio of $2: 1)($ Fig. $1 B$ ). The inhibitory effect of hASIC3 G439R was also measured when coexpressed with rodent ASIC1a (11.1 \pm 1.9 vs $1.5 \pm 0.7 \mathrm{nA}$ at a ratio of $2: 1)$ and ASIC2a $(4.5 \pm 0.6$ vs $0.8 \pm 0.2 \mathrm{nA}$ at a ratio $2: 1)$ subunits in transiently transfected HEK293 cells (Fig. 1C). Constitutive activity was tested by measuring the leak currents in COS-7 cells transiently transfected with hASIC3 G439R and by comparing with leak currents in COS-7 cells transiently transfected with wild-type hASIC3. The small leak currents in cells transfected with the mutant ASIC3 (density, $2.3 \pm 0.6 \mathrm{pA} / \mathrm{pF}$ ) were not larger than the leak currents recorded in cells transfected with the wild-type subunits (density, $3.0 \pm 0.7 \mathrm{pA} / \mathrm{pF})(n=$ 7-10). Moreover, we did not observe a blockade of basal constitutive currents by the antagonist amiloride applied at $100 \mu \mathrm{M}$ in cells expressing hASIC3 G439R (data not shown). These data show that G439R is a loss-of-function mutation for ASIC3. The hASIC3 G439R transgene expression, driven by the promoter of the neurofilament light chain gene (Fig. 2 A), was specifically detected in total brain and in DRGs of adult transgenic FVB mice through the identification of an exogenous $1.9 \mathrm{~kb}$ mRNA in Northern blot analysis (Fig. $2 \mathrm{~B}$ ) and the amplification of a $400 \mathrm{bp}$ product in RT-PCR (Fig. 2C), respectively.

DRG neurons from ASIC transgenic mice lack proton-evoked transient inward currents

To assess whether expression of the dominant-negative ASIC3 G439R subunit alters ASIC-like transient proton currents, we examined the responsiveness of acutely isolated DRG neurons to proton stimuli ( $\mathrm{pH}$ 5.0). A pH value of 5.0 was chosen because this concentration of protons induces maximal activation of the cloned ASIC3 channel current (Anzai et al., 2002; Benson et al., 2002). Neurons of all soma sizes were examined (diameter range, 15.5-43.2 $\mu \mathrm{m}$ ). Responses of neurons from wild-type mice to $\mathrm{pH}$ 5.0 fell into two classes as reported previously (Dirajlal et al., 2003). The first profile was a transient ASIC-like response that rapidly inactivated within $1-2 \mathrm{~s}$, and all of the transient responses were followed by a sustained current of $>100 \mathrm{pA}$ peak amplitude. The second profile was a sustained-only, slowly-inactivating inward current $(>20 \mathrm{~s})$. Figure $3 \mathrm{~A}$ shows a typical transient, fol-

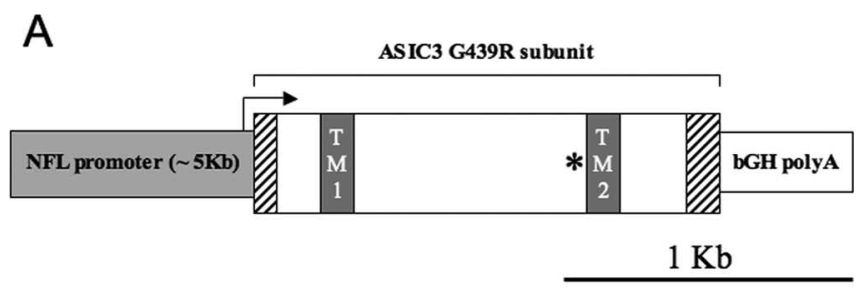

B

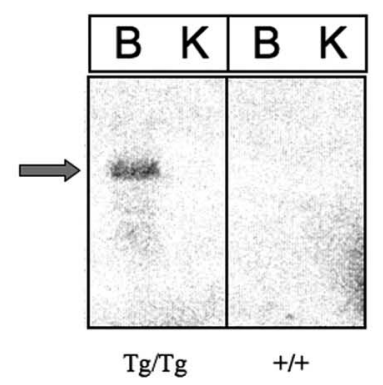

C



Figure 2. Transgenic expression of the ASIC3 G439R subunit in adult FVB mice. $\boldsymbol{A}$, Schematic organization of the transgene. The relative position of the mutation is indicated by the asterisk. $\boldsymbol{B}$, Northern blot showing NFL promoter-driven expression of ASIC3 G439R mRNA in the brain of homozygous transgenic animals. B, Whole brain; K, kidney. C, Detection of mutant ASIC3 mRNA $(M)$ in the brain and DRG isolated from homozygous transgenic animals using RT-PCR. Wildtype FVB mice were used as negative controls. Tg, Transgenic; NFL, neurofilament light chain.

lowed by sustained response and a typical sustained-only response of two different DRG neurons isolated from wild-type mice to a $10 \mathrm{~s}$ application of a $\mathrm{pH} 5.0$ solution. There was no difference in the percentage of neurons that responded to protons with an inward current (all profile types), because $45.7 \%$ of neurons from wild-type mice and $44.2 \%$ of neurons from transgenic mice responded to $\mathrm{pH} 5.0$ ( $p=1.0$; Fisher's exact test) (Fig. $3 B$ ). There were no differences in the mean diameter of neurons tested with protons between the two genotypes (wild type, $29 \pm$ 
A

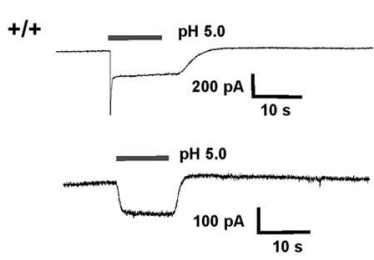

$\mathrm{Tg} / \mathrm{Tg}$

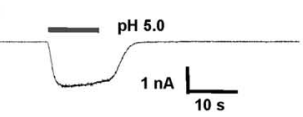

C

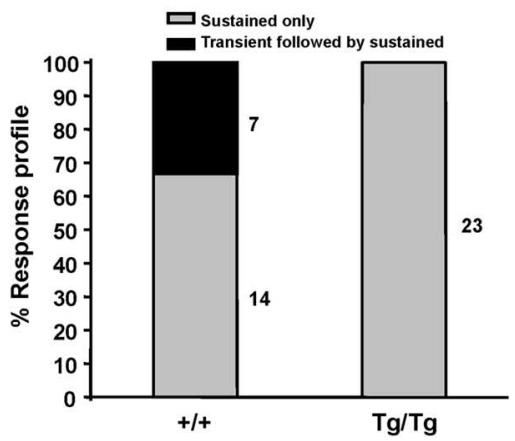

$\mathrm{B}$


Figure 3. Responses of DRG neurons from wild-type and transgenic mice to protons. $A$, Examples of inward current evoked by $\mathrm{pH} 5.0$ in two different neurons from wild-type mice and one from a transgenic mouse. $\boldsymbol{B}$, Percentage of neurons responding to $\mathrm{pH}$ 5.0. The numbers inside the bars indicate the $n$ tested. $C$, Percentage of proton-evoked responses that were transient, followed by a sustained or sustained-only current. $\boldsymbol{D}$, The bars represent the mean \pm SEM average peak magnitude of proton-evoked total current (open bars), sustained-only current (gray bars), transient-only current (black bar), and sustained after transient current (hatched bar) in DRG neurons from wild-type and transgenic mice. Tg, Transgenic.

A

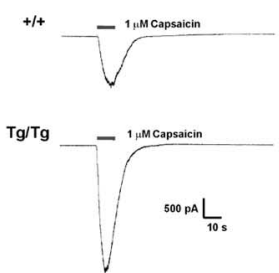

B

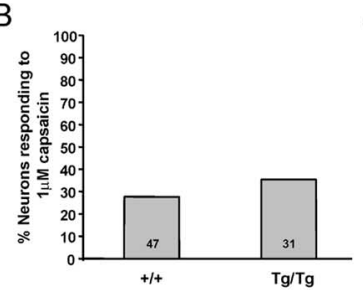

C

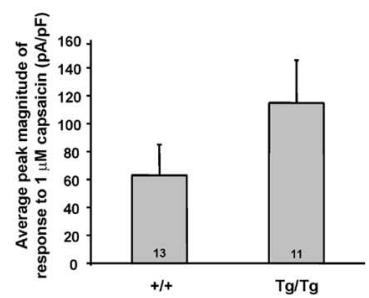

Figure 4. Responses of DRG neurons from wild-type and transgenic mice to $1 \mu \mathrm{m}$ capsaicin. $\boldsymbol{A}$, Examples of inward current evoked by $1 \mu \mathrm{m}$ capsaicin in neurons from a wild-type and transgenic mouse. $\boldsymbol{B}$, Percentage of neurons responding to $1 \mu \mathrm{M}$ capsaicin. The numbers inside the bars indicate the $n$ tested. $\boldsymbol{C}$, The bars represent the mean \pm SEM peak magnitude of capsaicinevoked inward current in DRG neurons from wild-type and transgenic mice. The numbers inside the bars indicate the $n$ that responded. Tg, Transgenic.

$1.0 \mu \mathrm{m}$; transgenic, $27 \pm 0.7 \mu \mathrm{m})$. Furthermore, there was no difference in the baseline leak current between the two genotypes (wild type: $58.2 \pm 9.1 \mathrm{pA}, n=46$; transgenic: $53.4 \pm 10.1 \mathrm{pA}, n=$ $52 ; p=0.73 ; t$ test), indicating that there is no constitutive current in the neurons from ASIC3 dominant-negative mutants. The profile of proton-evoked responses was significantly different between neurons from ASIC3 dominant-negative mutants and wild-type mice. Whereas $33 \%$ of the protonresponsive neurons from wild-type mice exhibited an ASIClike transient response, none of the neurons from the ASIC transgenic mice exhibited a transient inward current $(p<$ 0.005; Fisher's exact test) (Fig. 3C). There was no difference in the average peak magnitude of the sustained-only current in wild-type mice $(16.4 \pm 4.4 \mathrm{pA} / \mathrm{pF})$ and transgenic mice $(17.9 \pm 4.8 \mathrm{pA} / \mathrm{pF})$. This indicates that only the ASIC-like transient component of the proton-induced responses is altered by the ASIC3 dominant-negative mutation.
Capsaicin-evoked responses are unaltered in ASIC transgenic mice

To determine whether other protongated, non-ASIC family channels are affected by the ASIC3 dominant-negative mutation, we examined responses of neurons to capsaicin, a selective agonist for the TRPV1 receptor. TRPV1 is a member of the transient receptor potential (TRP) family, is localized to small- and mediumdiameter nociceptive DRG neurons, and constitutes a nonselective cation channel that can be activated by several natural and endogenous stimuli including protons (Caterina et al., 1997; Tominaga et al., 1998). Figure $4 A$ shows typical responses of DRG neurons from wild-type and transgenic mice. There was no difference in the percentage of capsaicin-responsive neurons between the two genotypes, because $27.7 \%$ of neurons from wild-type mice and $35.5 \%$ of neurons from transgenic mice responded to $1 \mu \mathrm{M}$ capsaicin (Fig. $4 B)(p=0.62$; Fisher's exact test). There were no differences in the mean diameter of neurons tested with capsaicin between the two genotypes (wild type, $23.7 \pm 0.8 \mu \mathrm{m}$; transgenic, $22.5 \pm 0.7$ $\mu \mathrm{m})$. Although there was a trend for the capsaicin-evoked current to be larger in the ASIC3 dominant-negative mutants, it was not statistically significant (Fig. 4C) (wild type, $63.1 \pm 21.9 \mathrm{pA} / \mathrm{pF}$; transgenic, $114.9 \pm 30.6 \mathrm{pA} / \mathrm{pF} ; p=0.21$; unpaired $t$ test). Therefore, these data indicate that proton-sensitive TRPV1 channels are unaffected by the ASIC3 dominant-negative subunit.

\section{Algesiometric assays}

Results from these assays are presented chronologically, in the order the data were collected.

\section{Tail-withdrawal test}

As shown in Figure $5 A$, wild-type and transgenic mice displayed statistically equivalent average latencies to withdraw the tail from $47^{\circ} \mathrm{C}$ water $\left(t_{(19)}=1.1 ; p=0.29\right)$ or $49^{\circ} \mathrm{C}$ water $\left(t_{(24)}=0.7 ; p=0.48\right)$.

\section{Hot-plate test}

As shown in Figure 5B, wild-type and transgenic mice displayed statistically equivalent latencies to perform a nocifensive response on a hot plate set at $50^{\circ} \mathrm{C}\left(t_{(21)}=2.1 ; p=0.05\right)$ or $53^{\circ} \mathrm{C}$ $\left(t_{(27)}=1.3 ; p=0.20\right)$.

Paw-withdrawal test

As shown in Figure 5C, wild-type and transgenic mice displayed statistically equivalent average latencies to withdraw from radiant heat source set at $10 \%\left(t_{(19)}=0.2 ; p=0.87\right)$ or $20 \%\left(t_{(21)}=1.2\right.$; $p=0.25)$ of maximal intensity.

Together, results from these three assays indicate that behavioral responses to acute noxious heat are not affected by the ASIC3 dominant-negative mutation. 
von Frey test

As shown in Figure 5D, a significantly lower threshold force was required to elicit withdrawal responses in transgenic mice compared with wild-type mice $\left(t_{(19)}=2.7\right.$; $p<0.05)$.

\section{Tail-clip test}

As shown in Figure 5E, transgenic mice displayed a significantly lower latency to remove the noxious tail clip than wild-type mice $\left(t_{(56)}=2.2 ; p<0.05\right)$.

Results from the von Frey and tail-clip tests indicate that the ASIC3 dominantnegative mutation increases sensitivity to mechanical stimuli.

\section{Abdominal constriction test}

As shown in Figure 5F, transgenic mice displayed significantly more total abdominal constrictions than wild-type mice after injection of $0.6 \%$ acetic acid $\left(t_{(10)}=\right.$ 3.8; $p<0.005)$ but not $0.3 \%\left(t_{(8)}=0.7\right.$; $p=0.50)$ or $0.9 \%\left(t_{(18)}=0.2 ; p=0.81\right)$ acetic acid.

\section{Formalin test}

As shown in Figure $5 G$, transgenic mice displayed significantly more licking/biting behavior than wild-type mice in both the acute $\left(t_{(16)}=2.8 ; p=0.01\right)$ and tonic $\left(t_{(16)}=2.3 ; p<0.05\right)$ phases of the $5 \%$ formalin test. Measurements of cross-sectional hindpaw thickness immediately after the conclusion of the formalin test revealed no genotype differences in hindpaw inflammation $\left(t_{(16)}=0.3 ; p=0.77\right)$.

Results from the abdominal constriction and formalin test indicate that the ASIC3 dominant-negative mutation increases sensitivity to inflammatory stimuli under certain conditions.

\section{Zymosan hypersensitivity}

For both thermal and mechanical hypersensitivity induced by zymosan administration, repeated-measures ANOVAs confirmed that both genotypes displayed significant and robust hypersensitivity, fully developed by $120 \mathrm{~min}$ after injection and stable out to $360 \mathrm{~min}$ (Fig. 6). A repeated-measures ANOVA performed on thermal sensitivity data (averaged baseline data and all post-injection time points; ipsilateral paw) revealed only a significant main effect of repeated measure $\left(F_{(6,54)}=8.7 ; p<\right.$ $0.001)$; the main effect of genotype and genotype-by-repeated measure interaction were nonsignificant. There was no significant effect of repeated measures for data from the contralateral hindpaw.

A repeated-measures ANOVA performed on mechanical sensitivity data (baseline and all post-injection time points; ipsilateral hindpaw) revealed a significant main effect of repeated measure $\left(F_{(6,156)}=29.5 ; p<0.001\right)$ and a significant genotypeby-repeated measure interaction $\left(F_{(6,156)}=2.4 ; p<0.05\right)$. Student's $t$ tests comparing genotype response frequencies at individual time points revealed that transgenic mice displayed significantly greater mechanical hypersensitivity than wild types at 180 and 240 min after injection $(p<0.05)$; genotype differences approached significance at other post-injection time points (Fig. 6). Both genotypes displayed contralateral mechanical hypersensitivity as well (data not shown), as evidenced by a significant effect of repeated measures, but there was no significant interaction of genotype by repeated measure for the contralateral paw.
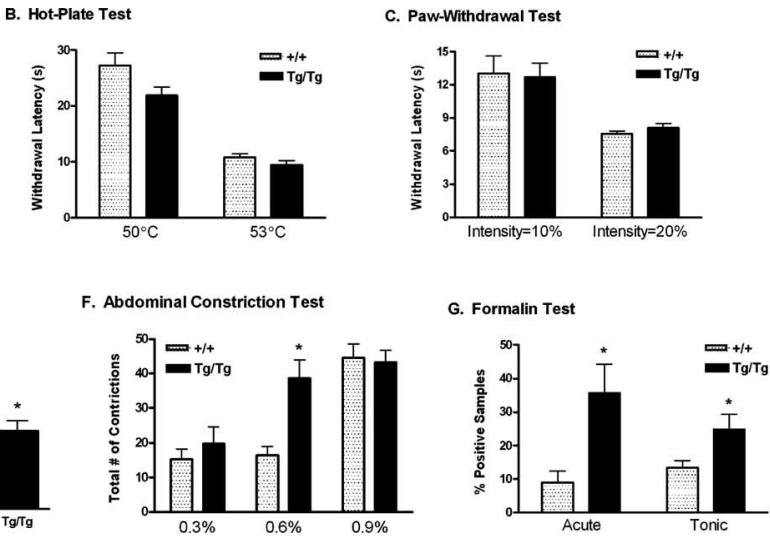

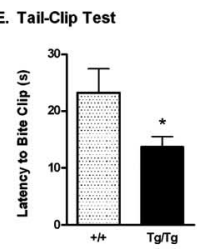

Figure 5. Sensitivity of wild-type $(+/+)$ and transgenic $(\mathrm{Tg} / \mathrm{Tg})$ mice on a variety of acute and tonic nociceptive assays. See (hethods for experimental details. The bars represent the following: $\boldsymbol{A}-\boldsymbol{C}$, mean \pm SEM latency to withdraw from $\operatorname{clip}$ (in seconds); $\boldsymbol{F}$, mean \pm SEM total number of abdominal constrictions; $\boldsymbol{G}$, mean \pm SEM percentages of 5 s samples showing licking 列

A.

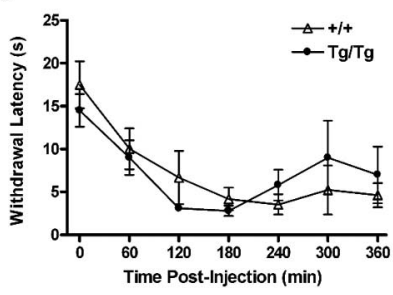

B.

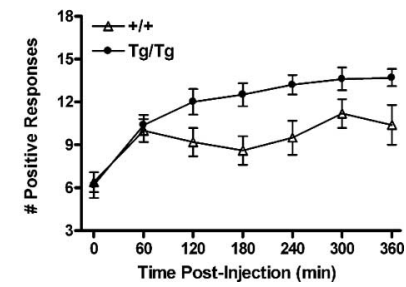

Figure 6. Increased mechanical, but not thermal, hypersensitivity in transgenic $(\mathrm{Tg} / \mathrm{Tg})$ mice compared with wild-type mice $(+/+)$ after injection of $1 \mathrm{mg} / \mathrm{ml}$ zymosan into the right hindpaw. See Materials and Methods for experimental details. $\boldsymbol{A}$, Symbols represent the mean \pm SEM latency to withdraw from a thermal stimulus (paw-withdrawal test). $\boldsymbol{B}$, Symbols represent the mean \pm SEM total number of positive withdrawal responses to three von Frey fibers (\#5, \#7, and \#9; each applied 6 times). ${ }^{*} p<0.05$ compared with $+/+$ on the same assay.

Thermal latencies and mechanical withdrawal responses over the entire 360 min time course were also converted to areas over and under the curve, respectively. No difference was observed between genotypes with respect to overall thermal hypersensitivity (wild type, $63.4 \pm 13.8$; transgenic, $59.5 \pm 5.5 ; t_{(9)}=0.3$ ). In contrast, mutant mice exhibited significantly greater overall mechanical hypersensitivity (wild type, $33.9 \pm 6.4$; transgenic, $\left.52.2 \pm 4.8 ; t_{(20)}=2.3 ; p<0.05\right)$.

Thus, just as transgenic mice were found to be more sensitive to acute mechanical but not acute thermal stimuli, they appear to be more sensitive to mechanical but not thermal hypersensitivity after inflammatory insult.

\section{Intramuscular hypotonic saline test}

As predicted by the data shown in Figure $5 D$, transgenic mice were more sensitive than wild-type mice to the $\# 5$ von Frey fiber at baseline $\left(t_{(11)}<0.05\right)$. A repeated-measures ANOVA performed on data at all time points from the ipsilateral hindpaw revealed significant main effects of genotype $\left(F_{(1,11)}=22.2 ; p<\right.$ $0.001)$ and repeated measure $\left(F_{(7,77)}=16.0 ; p<0.001\right)$, and a significant genotype by repeated-measures interaction $\left(F_{(7,77)}=\right.$ 6.6; $p<0.001)$. Subsequent repeated-measures ANOVAs run separately on each genotype revealed a highly significant repeated-measures effect in transgenic mice $\left(F_{(7,42)}=26.9 ; p<\right.$ 0.001 ) but a nonsignificant repeated-measures effect in wild-type 
mice $\left(F_{(7,35)}=2.0 ; p=0.09\right)$. In the transgenic mice, mechanical sensitivity was significantly increased (after Sidak correction for multiple comparisons) relative to baseline at $1,5,6,8$, and $12 \mathrm{~d}$ after the first hypotonic saline injection. Data from the contralateral paw (data not shown) were almost identical to those from the ipsilateral paw. In an experiment run separately using C57BL/6 mice, we confirmed that this inbred strain, the one used by Sluka et al. (2003), did indeed display mechanical hypersensitivity after both injections.

\section{Discussion}

\section{Dominant-negative properties of ASIC3 G439R and ASIC currents in transgenic mice}

The single mutation G439R leading to inhibitory dominantnegative ASIC3 is located in the pre-TM2 region that has been shown to be critically involved in $\mathrm{pH}$ sensing, gating, and amiloride binding (Adams et al., 1999). The pre-TM2 region is highly conserved in all known channel subunits of the degenerin/ $\mathrm{ENaC1/ASIC}$ sodium channel superfamily (Kellenberger and Schild, 2002). Therefore, it was expected that substitution of a native glycine residue by a bulky and positively charged arginine residue would disrupt the function of the ASIC3 channel. The inhibitory effect of ASIC3 G439R subunits on ASIC1a and ASIC2a activity confirms heteromeric associations of ASIC3 subunits with ASIC1 and ASIC2, as observed previously in heterologous systems as well as in native preparations (Benson et al., 2002; Deval et al., 2004). The association of the ASIC3 G439R subunit with wild-type ASIC1, ASIC2, or ASIC3 subunits renders the resulting channels less sensitive to protons. Therefore, constitutive expression of exogenous ASIC3 G439R subunits in DRG neurons was designed to inhibit any type of fast proton-gated currents, whatever the native subunit composition of the channels mediating them.

Indeed, our recordings from DRGs isolated from transgenic mice revealed that all ASIC-like transient proton currents were completely inhibited. These data suggest that in mice, ASIC3 associates with both ASIC1 as well as ASIC2 in native DRG neurons and indicate that the dominant-negative mutation inhibits expression of all ASIC-like transient currents. In contrast, sustained-only proton-gated currents were unaltered in magnitude in the transgenic mice, indicating that sustained-only proton currents that may be mediated by TRPV1 (Tominaga et al., 1998), or proton-sensitive potassium channels such as TASK-1, TASK-3, or Kir2.3 (Baumann et al., 2004), are not affected by the dominant-negative subunit. Furthermore, we show that capsaicin-evoked inward currents were not affected by the dominant-negative subunit, further indicating that TRPV1 ion channels were unaltered in the transgenic mice.

\section{Algesiometric assays}

Behavioral testing of ASIC mutants on algesiometric assays has been performed on only three previous occasions, and significant phenotypic differences from wild-type mice have been shown only for ASIC3 knock-outs (Price et al., 2001; Chen et al., 2002; Sluka et al., 2003). The data in our study cannot be easily reconciled with any of these existing demonstrations; however, it must be borne in mind that the transgenic mice tested here were missing all ASIC-like transient currents (see above). Given that ASICs form heteromultimers in DRG neurons, a different phenotype in our transgenic "triple knock-out" compared with the ASIC3 knock-out might be expected.

\section{ASIC transgenic mice are unaltered in their behavioral responses to heat}

Price et al. (2001) found no differences in withdrawal latencies to thermal heat in the paw-withdrawal test. On the other hand, Chen et al. (2002) observed significantly reduced latencies in ASIC3 knock-outs in the hot-plate test using high plate temperatures, and they concluded that ASIC3 was involved in nociceptive processing at high stimulus intensities. However, we note that paw-withdrawal test data in the same study [Chen et al. (2002), their Fig. 4a] revealed no significant effects of the null mutation at a fairly intense stimulus (latencies $\approx 6-7 \mathrm{~s}$ ). With the hypothesis that ASIC effects might be intensity dependent in mind, we tested our transgenics using lower- and higherintensity heat stimuli on three different thermal assays and obtained no convincing evidence for any differences at either stimulus intensity. We conclude that if ASICs are indeed involved in the modulation of thermal nociception, their effects are subtle. Of course, it remains possible that proteins other than ASICs are fully compensating for the absence of ASIC currents in these mice.

\section{ASIC transgenic mice show increased behavioral sensitivity to mechanical stimuli}

With respect to mechanical nociception, neither Price et al. (2001) or Chen et al. (2002) observed any alterations in baseline mechanical sensitivity in ASIC3 knock-outs using a range of von Frey fibers. The fact that we did here might implicate ASIC1 or ASIC2 channels. However, it should also be noted that neither of the previous workers used the more sensitive up-down method (Chaplan et al., 1994) used in the experiment shown in Figure 5D. Using a response frequency criterion, we also failed to demonstrate genotype differences in baseline von Frey sensitivity in the zymosan experiment (Fig. 6), although we did observe them in the Sluka model experiment in which hypotonic saline is injected into muscle (Fig. 7). Our data using the tail-clip test is in agreement with the observation of Chen et al. (2002) of increased sensitivity to mechanical nociception on the tail-pressure test. Note that although our data appear to confirm the involvement of ASICs in the processing of mechanical stimuli, the direction of effect (transgenic mice more sensitive than wild-type mice) renders it quite unlikely that they are directly transducing mechanical stimuli.

\section{ASIC transgenic mice show increased sensitivity to chemical/inflammatory stimuli}

With respect to spontaneously emitted behavioral responses to chemical stimuli, Price et al. (2001) observed no differences in paw licking after an injection of acetic acid into the paw. Using a common assay, acetic acid-induced abdominal constriction, Chen et al. (2002) demonstrated significantly greater sensitivity in ASIC3 mutants, but only using $0.6 \%$ acetic acid. We also used multiple acetic acid concentrations $(0.3-0.9 \%)$ and also observed significant differences only at $0.6 \%$ (Fig. $5 F$ ). It is not at all clear why only the $0.6 \%$ concentration yielded significant genotype differences. We are confident of the general conclusion of increased sensitivity to chemical/inflammatory stimuli in the transgenic mice, however, because of the clear effect seen using a much more trustworthy assay (Wilson and Mogil, 2001), the formalin test. Again, the hypothesis that ASICs are involved in the processing of high-intensity stimuli is contradicted by our inability to observe a genotypic difference at $0.9 \%$, although it is possible that a ceiling effect is preventing this observation.

Our data showing the lack of influence of ASIC dysfunction 


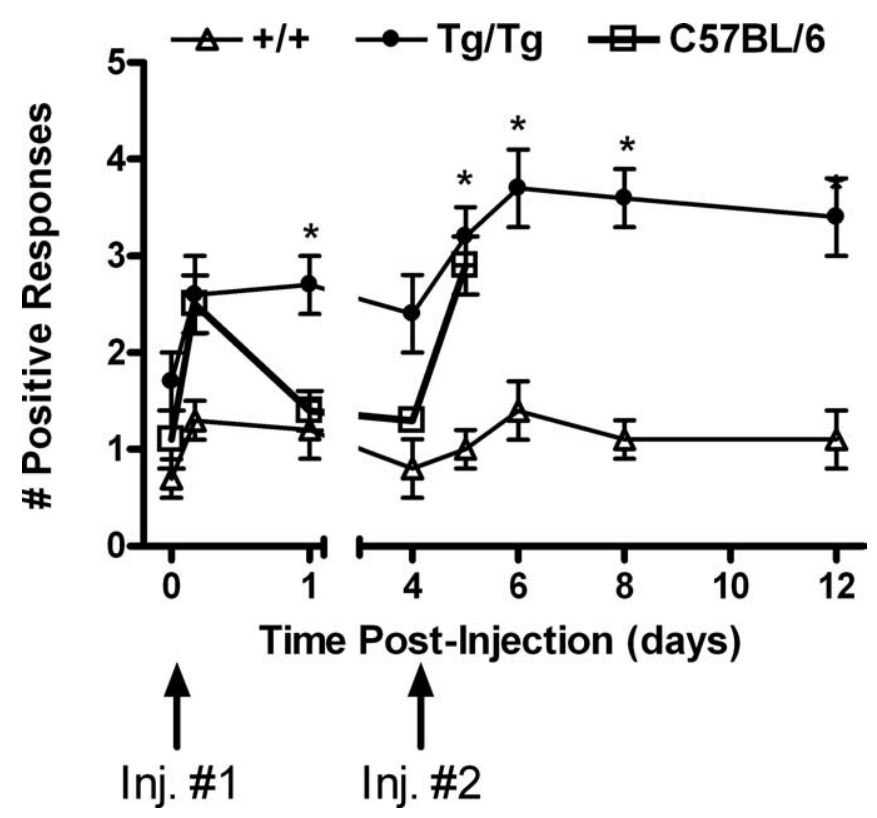

Figure 7. Mechanical hypersensitivity after intramuscular hypotonic saline injections [at day 0 (Inj. \#1) and day 4 (Inj. \#2)] in transgenic mice $(\mathrm{Tg} / \mathrm{Tg})$ but not wild-type mice $(+/+)$. See Materials and Methods for experimental details. The symbols represent the mean \pm SEM number of positive withdrawal responses to a \#5 von Frey fiber of the ipsilateral hindpaw. Responses of the contralateral hindpaw (data not shown) were almost identical. In a separate experiment using C57BL/6 mice, we confirmed the hypersensitivity of this strain as reported originally. ${ }^{*} p<0.05$ compared with baseline ( $\mathrm{Tg} / \mathrm{Tg}$ mice).

on thermal hypersensitivity produced by zymosan is in complete agreement with previous investigations, which both used carrageenan as the inflammatory stimulus (Price et al., 2001; Chen et al., 2002). Our observation of significantly enhanced mechanical hypersensitivity from zymosan is in agreement with those of Price et al. (2001) using carrageenan but in contrast to those of Chen et al. (2002), who observed equivalent mechanical hypersensitivity in both genotypes after carrageenan and capsaicin. It should be noted, though, that these inflammatory substances produced such robust hypersensitivity in their hands [Chen et al. (2002), their Fig. 4b,c] that it may not have been possible to measure any increase from wild-type levels.

Finally, our data using intramuscular hypotonic saline injections contradict those of Sluka and colleagues (Price et al., 2001; Sluka et al., 2003), in that they observed a blockade of mechanical hypersensitivity in ASIC3 knock-outs, whereas we see an enhancement in transgenic mice. The likeliest explanation for this discrepancy is genetic background, because Sluka et al. (2003) tested knock-out mice on a C57BL/6 background, whereas our transgenic mice were constructed on an FVB background. We confirmed here that C57BL/6 mice do become hypersensitive to mechanical stimulation after intramuscular hypotonic saline injections, but apparently FVB mice do not. This is not as surprising as it may seem, because robust strain dependence of mechanical hypersensitivity has been demonstrated repeatedly (Mogil et al., 1999; Smith et al., 2004; LaCroix-Fralish et al., 2005). It should be noted that background genotype may also be influencing other discrepancies noted above, because the mutants in the study by Price et al. (2001) were on an $(129 \times \mathrm{C} 57 \mathrm{BL} / 6) \mathrm{F}_{2}$ background, whereas the mutants in the study by Chen et al. (2002) were on a $(\mathrm{CD}-1 \times \mathrm{C} 57 \mathrm{BL} / 6) \mathrm{F}_{2}\left(\right.$ or $\left.\mathrm{F}_{4}\right)$ background.

Native ASIC3 subunits are associated with the multivalent PDZ (postsynaptic density-95/Discs large/zona occludens-1)- binding domain protein CIPP, which modulates their surface expression (Anzai et al., 2002), so overexpression of exogenous ASIC3 subunits could lead to abnormal trafficking of CIPP or other unknown ASIC3 partners involved in sensory transduction. Thus, compensatory changes in pronociceptive pathways, central or peripheral, could also explain the hypersensitivity of the transgenic mice to noxious stimuli despite the suppression of ASIC currents in the DRG neurons. It is also possible that the phenotypes observed here may be related to direct participation of hippocampal ASICs or spinal cord ASICs in pain processing. Finally, although less likely, it is also possible that the channels containing mutant ASIC3 subunits, tested for the absence of response to protons, remain sensitive to other unidentified agonists that play a role in nociception. The expression of ASIC2 has been reported recently to protect against light-induced neurodegeneration in the retina (Ettaiche et al., 2004), and the knock-out of ASIC1 leads to hypersensitivity of visceral mechanoreceptors (Page et al., 2004). The hypersensitivity of transgenic animals expressing dominant-negative ASIC3 subunits to mechanical and inflammatory stimuli points also to a regulatory role for ASICs in sensory pathways.

\section{References}

Adams CM, Snyder PM, Welsh MJ (1999) Paradoxical stimulation of a DEG/ENaC channel by amiloride. J Biol Chem 274:15500-15504.

Alvarez de la Rosa D, Zhang P, Shao D, White F, Canessa CM (2002) Functional implications of the localization and activity of acid-sensitive channels in rat peripheral nervous system. Proc Natl Acad Sci USA 99:2326-2331.

Anzai N, Deval E, Schaefer L, Friend V, Lazdunski M, Lingueglia E (2002) The multivalent PDZ domain-containing protein CIPP is a partner of acid-sensing ion channel 3 in sensory neurons. J Biol Chem 277:16655-16661.

Babinski K, Le KT, Seguela P (1999) Molecular cloning and regional distribution of a human proton receptor subunit with biphasic functional properties. J Neurochem 72:51-57.

Baumann TK, Chaudhary P, Martenson ME (2004) Background potassium channel block and TRPV1 activation contribute to proton depolarization of sensory neurons from humans with neuropathic pain. Eur J Neurosci 19:1343-1351.

Benson CJ, Xie J, Wemmie JA, Price MP, Henss JM, Welsh MJ, Snyder PM (2002) Heteromultimers of DEG/ENaC subunits form $\mathrm{H}^{+}$-gated channels in mouse sensory neurons. Proc Natl Acad Sci USA 99:2338-2343.

Breese NM, George AC, Pauers LE, Stucky CL (2005) Peripheral inflammation selectively increases TRPV1 function in IB4-positive sensory neurons from adult mouse. Pain 115:37-49.

Caterina MJ, Schumacher MA, Tominaga M, Rosen TA, Levine JD, Julius D (1997) The capsaicin receptor: a heat-activated ion channel in the pain pathway. Nature 389:816-824.

Chaplan SR, Bach FW, Pogrel JW, Chung JM, Yaksh TL (1994) Quantitative assessment of tactile allodynia evoked by unilateral ligation of the fifth and sixth lumbar nerves in the rat. J Neurosci Methods 53:55-63.

Charron G, Guy LG, Bazinet M, Julien JP (1995) Multiple neuron-specific enhancers in the gene coding for the human neurofilament light chain. J Biol Chem 270:30604-30610.

Chen C-C, Zimmer A, Sun W-H, Hall J, Brownstein MJ, Zimmer A (2002) A role for ASIC3 in the modulation of high-intensity pain stimuli. Proc Natl Acad Sci USA 99:8992-8997.

Chesler EJ, Ritchie J, Kokayeff A, Lariviere WR, Wilson SG, Mogil JS (2003) Genotype-dependence of gabapentin and pregabalin sensitivity: the pharmacogenetic mediation of analgesia is specific to the type of pain being inhibited. Pain 106:325-335.

Deval E, Salinas M, Baron A, Lingueglia E, Lazdunski M (2004) ASIC2bdependent regulation of ASIC3, an essential acid-sensing ion channel subunit in sensory neurons via the partner protein PICK-1. J Biol Chem 279:19531-19539.

Dirajlal S, Pauers LE, Stucky CL (2003) Differential response properties of $\mathrm{IB}(4)$-positive and -negative unmyelinated sensory neurons to protons and capsaicin. J Neurophysiol 89:513-524. 
Drew LJ, Rohrer DK, Price MP, Blaver KE, Cockayne DA, Cesare P, Wood JN (2004) Acid-sensing ion channels ASIC2 and ASIC3 do not contribute to mechanically activated currents in mammalian sensory neurones. J Physiol (Lond) 556:691-710.

Ettaiche M, Guy N, Hofman P, Lazdunski M, Waldmann R (2004) Acidsensing ion channel 2 is important for retinal function and protects against light-induced retinal degeneration. J Neurosci 24:1005-1012.

Hargreaves K, Dubner R, Brown F, Flores C, Joris J (1988) A new and sensitive method for measuring thermal nociception in cutaneous hyperalgesia. Pain 32:77-88.

Kellenberger S, Schild L (2002) Epithelial sodium channel/degenerin family of ion channels: a variety of functions for a shared structure. Physiol Rev 82:735-767.

Krishtal OA, Pidoplichko VI (1980) A receptor for protons in the nerve cell membrane. Neuroscience 5:2325-2327.

LaCroix-Fralish ML, Mogil JS, Weinstein JN, Rutkowski MD, DeLeo JA (2005) The magnitude of mechanical allodynia in a rodent model of lumbar radiculopathy is dependent on strain and sex. Spine 30:1821-1827.

Lariviere WR, Wilson SG, Laughlin TM, Kokayeff A, West EE, Adhikari SM, Wan Y, Mogil JS (2002) Heritability of nociception. III. Genetic relationships among commonly used assays of nociception and hypersensitivity. Pain 97:75-86.

Mamet J, Baron A, Lazdunski M, Voilley N (2002) Proinflammatory mediators, stimulators of sensory neuron excitability via the expression of acid-sensing ion channels. J Neurosci 22:10662-10670.

Meller ST, Gebhart GF (1997) Intraplantar zymosan as a reliable, quantifiable model of thermal and mechanical hyperalgesia in the rat. Eur J Pain 1:43-52.

Mogil JS, Wilson SG, Bon K, Lee SE, Chung K, Raber P, Pieper JO, Hain HS, Belknap JK, Hubert L, Elmer GI, Chung JM, Devor M (1999) Heritability of nociception. I. Responses of eleven inbred mouse strains on twelve measures of nociception. Pain 80:67-82.

O'Hagan R, Chalfie M, Goodman MB (2005) The MEC-4 DEG/ENaC channel of Caenorhabditis elegans touch receptor neurons transduces mechanical signals. Nat Neurosci 8:43-50.

Page AJ, Brierley SM, Martin CM, Martinez-Salgado C, Wemmie JA, Brennan TJ, Symonds E, Omari T, Lewin GR, Welsh MJ, Blackshaw LA (2004) The ion channel ASIC1 contributes to visceral but not cutaneous mechanoreceptor function. Gastroenterology 127:1739-1747.
Price MP, Lewin GR, McIlwrath SL, Cheng C, Xie J, Heppenstall PA, Stucky CL, Mannsfeldt AG, Brennan TJ, Drummond HA, Qiao J, Benson CJ, Tarr DE, Hrksta RF, Yang B, Williamson RA, Welsh MJ (2000) The mammalian sodium channel BNC1 is required for normal touch sensation. Nature 407:1007-1011.

Price MP, McIlwrath SL, Xie J, Cheng C, Qiao J, Tarr DE, Sluka KA, Brennan TJ, Lewin GR, Welsh MJ (2001) The DRASIC cation channel contributes to the detection of cutaneous touch and acid stimuli in mice. Neuron 32:1071-1083.

Reeh PW, Steen KH (1996) Tissue acidosis in nociception and pain. Prog Brain Res 113:143-151.

Roza C, Puel JL, Kress M, Baron A, Diochot S, Lazdunski M, Waldmann R (2004) Knockout of the ASIC2 channel in mice does not impair cutaneous mechanosensation, visceral mechanonociception and hearing. J Physiol (Lond) 558:659-669.

Shu X, Mendell LM (2001) Acute sensitization by NGF of the response of small-diameter sensory neurons to capsaicin. J Neurophysiol 86:2931-2938.

Sluka KA, Price MP, Breese NM, Stucky CL, Wemmie JA, Welsh MJ (2003) Chronic hyperalgesia induced by repeated acid injections in muscle is abolished by the loss of ASIC3, but not ASIC1. Pain 106:229-239.

Smith SB, Crager SE, Mogil JS (2004) Paclitaxel-induced neuropathic hypersensitivity in mice: responses in 10 inbred mouse strains. Life Sci 74:2593-2604.

Tominaga M, Caterina MJ, Malmberg AB, Rosen TA, Gilbert H, Skinner K, Raumann BE, Basbaum AI, Julius D (1998) The cloned capsaicin receptor integrates multiple pain-producing stimuli. Neuron 21:531-543.

Voilley N, de Weille J, Mamet J, Lazdunski M (2001) Nonsteroid antiinflammatory drugs inhibit both the activity and the inflammationinduced expression of acid-sensing ion channels in nociceptors. J Neurosci 21:8026-8033.

Waldmann R, Champigny G, Bassilana F, Heurteaux C, Lazdunski M (1997) A proton-gated cation channel involved in acid-sensing. Nature 386:173-177.

Wilson SG, Mogil JS (2001) Measuring pain in the (knockout) mouse: big challenges in a small mammal. Behav Brain Res 125:65-73.

Winter J, Forbes CA, Sternberg J, Lindsay RM (1988) Nerve growth factor (NGF) regulates adult rat cultured dorsal root ganglion neuron responses to the excitotoxin capsaicin. Neuron 1:973-981. 